\title{
Mechanical stress affects the osteogenic differentiation of human ligamentum flavum cells via the BMP-Smad1 signaling pathway
}

\author{
SHUNZHI YU ${ }^{1 *}$, ZHONGHAI LI $^{2 *}$ and NING YAN ${ }^{1}$ \\ ${ }^{1}$ Department of Orthopedic Surgery, Shanghai Tenth People's Hospital, Tongji University School of Medicine, \\ Shanghai 200072; ${ }^{2}$ Department of Orthopedics, First Affiliated Hospital of \\ Dalian Medical University, Dalian 116011, P.R. China
}

Received December 25, 2015; Accepted February 14, 2017

DOI: $10.3892 / \mathrm{mmr} .2017 .7543$

\begin{abstract}
The aim of the present study was to investigate the effects of mechanical stress on the osteogenic differentiation of human ligamentum flavum cells via the bone morphogenetic protein (BMP)-Smad1 signaling pathway. Mechanical stress increased cell proliferation and induced osteogenic differentiation of human cells derived from the ossification of the ligamentum flavum (OLF). In addition, mechanical stress activated osteocalcin (OC), alkaline phosphatase (ALP) and runt-related transcription factor 2 (RUNX-2) mRNA expression, and suppressed Ets proto-oncogene 1 (Ets-1) and sex determining region Y-box 2 (SOX-2) mRNA expression in OLF cells. Src protein expression was suppressed by mechanical stress in human OLF cells. In addition, the protein expression levels of BMP, phosphorylated (p)-mothers against decapentaplegic homolog-1 (Smad1) and p-p38-mitogen-activated protein kinases (p38MAPK) were increased by mechanical stress. These results demonstrate that mechanical stress effectively increases cell proliferation, promotes the osteogenic differentiation rate of OLF cells, activates OC, ALP and RUNX-2, and suppresses Ets-1 and SOX-2 potentially via the BMP-Smad1 and Src-p38MAPK signaling pathways.
\end{abstract}

\section{Introduction}

Ossification of the ligamentum flavum (OLF) is the most common etiology of thoracic spinal stenosis (1). Although the causes and pathogeneses are unclear, OLF is thought to

Correspondence to: Dr Ning Yan, Department of Orthopedic Surgery, Shanghai Tenth People's Hospital, Tongji University School of Medicine, 301 Yan Chang Zhong Road, Shanghai 200072, P.R. China

E-mail: ningyanning123@yeah.net

${ }^{*}$ Contributed equally

Key words: mechanical stress, human ligamentum flavum cells, Src, bone morphogenetic protein, p38-mitogen-activated protein kinase, mothers against decapentaplegic homolog-1 occur due to genetic and environmental factors, including diet, humidness, metabolic disturbance and athletic injury (2). A number of surgical procedures are used to treat OLF in the clinic, including lamina eroding decompression, double-door lamina resection decompression, the whole vertebral plate decompression peel method, and lamina decompression and implant surgery (3). The lamina eroding decompression method is a relatively simple operation; however, the double-door and vertebral plate decompression methods require high-speed drills and osteotomes (1). The loss of the spinous process and vertebral plate weaken the stability of the posterior column and, after a significant period of time, may produce unstable regions of reduced pressure (4). To maintain the intact structure of the canalis vertebralis, lamina decompression and implant surgery are performed (5). Implanted vertebral plates ensure that the structure of the canalis spinalis is maintained and prevents scar tissue from invading the canalis spinalis (6). In addition, vertebral plates provide attachment points for paravertebral muscles and prevent amyotrophy. The fixation of miniature titanium plates maintains the integrity of the spinal cord. However, when compared with laminectomies, vertebral plate implant surgery is more complex, with longer operating times and increased levels of bleeding (7).

Mechanical stress stimulation serves an important regulatory function in bone formation and remodeling (8). Mechanical stimuli target bone tissues and are transmitted as one of three local signals, which include fluid shear stress, cytomorphosis or stress-generated potentials (9). Cells sensitive to transformation stress transduce these local signals into primary signals via the following three pathways: The integrin-cytoskeleton system, the calcium channel pathway and the primary cilium pathway (10). These primary signals are subsequently transmitted as downstream signals via signal transduction pathways (11). These signals induce a number of alterations, including those associated with genetic expression, energy metabolism and material synthesis.

OLF is a common disease, which is characterized by heterotopic osteogenesis of the posterior longitudinal ligament of the cervical vertebra in China (12). Unregulated growth of an ossific mass in the canalis spinalis may lead to severe spinal cord and nerve root entrapment; however, the pathogenesis of OLF is currently unclear (13). The aim of the present study was to determine the effect of mechanical stress on the rate of 
osteogenic differentiation of human ligamentum flavum cells, and to elucidate the underlying mechanisms involved.

\section{Materials and methods}

Cell culture and generation of the mechanical stress model. Human OLF tissues (3-5 g) were obtained during surgery from a patient admitted to the Shanghai Tenth People's Hospital, Shanghai, China (aged 53 years; male; stage IV lumbar degenerative disease, during March 2015) that was diagnosed with lumbar degenerative disease. Written informed consent was provided by the patient for the use of their tissue samples in the present study. The tissues were cut into $1-2-\mathrm{mm}^{3}$ sections and digested with $2 \%$ type-I collagenase (Sunshine Biotechnology Co., Ltd., Nanjing, China) at $37^{\circ} \mathrm{C}$ for $30 \mathrm{~min}$. Nucleated cells $\left(10,000 \mathrm{cells} / \mathrm{cm}^{2}\right)$ were recovered and plated as mononuclear cells to obtain a higher yield. Cells were cultured in $\alpha$-minimum essential medium (Gibco; Thermo Fisher Scientific, Inc., Waltham, MA, USA) supplemented with $15 \%$ fetal bovine serum (Hyclone; GE Healthcare Life Sciences, Logan, UT, USA) at $37^{\circ} \mathrm{C}$ in $5 \%$ $\mathrm{CO}_{2}$; the medium was refreshed every 2 days. Following 1 week of culture, the cells were transferred to culture flasks coated with fibronectin $\left(5 \mu \mathrm{g} / \mathrm{cm}^{2}\right.$, Sigma-Aldrich; Merck KGaA, Darmstadt, Germany). A specially-designed four-point bending apparatus with flexible silicon-bottomed chambers was generated as described previously (14), and used to induce the mechanical stress model. The cells were then subjected to uniaxial tensile strain $(0.5 \mathrm{~Hz}, 2,000 \mathrm{~min}$, three times/day).

Cell viability. Cells were distributed into control, unstretched and stretched groups.

In the control group, cells were treated with PBS; in unstretched group, cells were subjected to uniaxial tensile strain without voltage; in stretched group, cells were subjected to uniaxial tensile strain $(0.5 \mathrm{~Hz}, 2,000 \mathrm{~min}$, three times/day).

Following 3 weeks of mechanical stress, cells $\left(1-2 \times 10^{3}\right)$ were cultured in a 96-well plate for $24 \mathrm{~h}$. A total of $20 \mu \mathrm{l}$ MTT assay reagent $(5 \mathrm{mg} / \mathrm{ml}$; Beyotime Institute of Biotechnology, Haimen, China) was then added to each well, and the plates were incubated at $37^{\circ} \mathrm{C}$ and $5 \% \mathrm{CO}_{2}$ for $4 \mathrm{~h}$. Following incubation, the culture medium was removed and $150 \mu$ l dimethyl sulfoxide (DMSO) was added to each well and allowed to dissolve the formazan crystals for $20 \mathrm{~min}$ at $37^{\circ} \mathrm{C}$. Cell viability was measured using a microplate reader (Omega Bio-Tek, Inc., Norcross, GA, USA) at a wavelength of $490 \mathrm{~nm}$.

Osteogenic differentiation. Cells $\left(1 \times 10^{5}\right)$ from the different groups were fixed in $95 \%$ ethanol overnight at $4^{\circ} \mathrm{C}$, and stained with Oil Red O $(0.25 \%)$ for $15 \mathrm{~min}$ at room temperature. The cells were then washed three times with $70 \%$ ethanol for $5 \mathrm{~min}$. Osteogenic differentiation was observed using a microscope in three field of view (Axio Imager 2; Zeiss GmbH, Jena, Germany).

Reverse transcription-quantitative polymerase chain reaction $(R T-q P C R)$. After 3 weeks mechanical stress, cells $\left(1 \times 10^{6}\right)$ from the different groups were cultured on a 6-well plate for $24 \mathrm{~h}$. Total RNA was isolated using TRIzol reagent (Invitrogen;
Thermo Fisher Scientific, Inc.) according to the manufacturer's instructions. A total of 2 ng RNA was reverse-transcribed into cDNA using a High-Capacity cDNA Reverse Transcription kit (Applied Biosystems; Thermo Fisher Scientific, Inc.) according to the manufacturer's instructions. For qPCR analysis, $1 \mathrm{ng}$ cDNA template was used for each reaction and sequences were amplified using a StepOne ${ }^{\mathrm{TM}}$ Real-Time PCR system and Power SYBR ${ }^{\circledR}$ Green PCR Master Mix (both from Applied Biosystems; Thermo Fisher Scientific, Inc.). Amplification reactions were conducted with an initial step at $95^{\circ} \mathrm{C}$ for $1 \mathrm{~min}$, followed by 40 cycles of $55^{\circ} \mathrm{C}$ for $30 \mathrm{sec}$ and $60^{\circ} \mathrm{C}$ for $30 \mathrm{sec}$. The primers used for qPCR analysis are listed in Table I and gene expression was normalized to the levels of $\beta$-actin and analyzed using the $2^{-\Delta \Delta C q}$ method (15).

Western blot analysis. Cells $\left(1 \times 10^{6}\right)$ after 3 weeks mechanical stress from the different groups were lysed with ice-cold lysis buffer containing a $10 \mu \mathrm{g} / \mathrm{ml}$ of protein inhibitor mixture (1:100; Roche Diagnostics, Indianapolis, IN, USA). Protein concentrations were then determined using a DC Protein assay kit (Bio-Rad Laboratories, Inc., Hercules, CA, USA). A total of $50 \mu \mathrm{g}$ protein was separated by $8-12 \%$ SDS-PAGE and electrophoretically transferred onto $0.22 \mu \mathrm{m}$ polyvinylidenedifluoride membranes (EMD Millipore, Billerica, MA, USA).The membranes were then washed with5\% non-fat dried milk in Tris-buffered solution plus $0.05 \%$ Tween- 20 (TBST) for $2 \mathrm{~h}$, prior to incubation with anti-Src (catalog no. sc-8995, dilution, 1:200), anti-bone morphogenetic protein (catalog no. sc-9003, BMP; dilution, 1:200) (both from Santa Cruz Biotechnology, Inc., Dallas, TX, USA), anti-phosphorylated (p)-mothers against decapentaplegic homolog-1 (catalog no. 13820, p-Smad-1; dilution, 1:400; Cell Signaling Technology, Inc., Danvers, MA, USA), anti-p-p38-mitogen-activated protein kinases (catalog no. sc-7975-R, p38MAPK; dilution, 1:300) and anti- $\beta$-actin (catalog no. sc-7210, dilution, 1:400) (both from Santa Cruz Biotechnology, Inc.) at $4^{\circ} \mathrm{C}$ overnight. The membranes were washed with TBST for $5 \mathrm{~min}$ three times and were subsequently incubated with anti-rabbit horseradish peroxidase-conjugated secondary antibody (catalog no. A0208, dilution, 1:4,000; Beyotime Institute of Biotechnology) at room temperature for $1 \mathrm{~h}$, and detected with electrochemiluminescence western blot detection reagents (Beyotime Institute of Biotechnology). Protein expression was analyzed using Quantity One software version 3.0 (Bio-Rad Laboratories, Inc., Hercules, CA, USA).

Statistical analysis. All data are expressed as the mean \pm standard deviation, and represent the mean values of at least three independent experiments using SPSS software version 17.0 (SPSS, Inc., Chicago, IL, USA). Comparisons among groups were analyzed using one-way analysis of variance, followed by the Tukey Kramer post hoc test. $\mathrm{P}<0.05$ was considered to indicate a statistically significant difference.

\section{Results}

Cell morphology. OLF cells were extracted using a fibronectin differential-adhesion assay, and OLF cell morphology was observed following 1 week of culture. As shown in Fig. 1, OLF cells exhibited a uniform morphology and were well-distributed 
Table I. Primer sequences used to analyzetarget gene expression levels by reverse transcription-quantitative polymerase chain reaction.

\begin{tabular}{lll}
\hline Gene & \multicolumn{1}{c}{ Forward sequence (5'-3') } & \multicolumn{1}{c}{ Reverse sequence (5'-3') } \\
\hline OC & ATGAGAGCCCTCACACTCCTC & GCCGTAGAAGCGCCGATAGGC \\
ALP & TGGAGCTTCAGAAGCTCAACACCA & ATCTCGTTGTCTGAGTACCAGTCC \\
RUNX-2 & ATCTCGTTGTCTGAGTACCAGTCC & ATCTCGTTGTCTGAGTACCAGTCC \\
SOX-2 & CCCCCCTGTGGTTACCTCTTC & CCCCCCTGTGGTTACCTCTTC \\
Ets-1 & GAGTTCAGCCTGAAGGGTGT & CACATCCTCTTTCTGCAGGATCT \\
$\beta$-actin & GTGGGGCGCCCCAGGCACCA & CTTCCTTAATGTCACGCACGATTTC
\end{tabular}

OC, osteocalcin; ALP, alkaline phosphatase; RUNX-2, runt-related transcription factor 2; SOX-2, sex determining region Y-box 2; Ets-1, Ets proto-oncogene 1 .

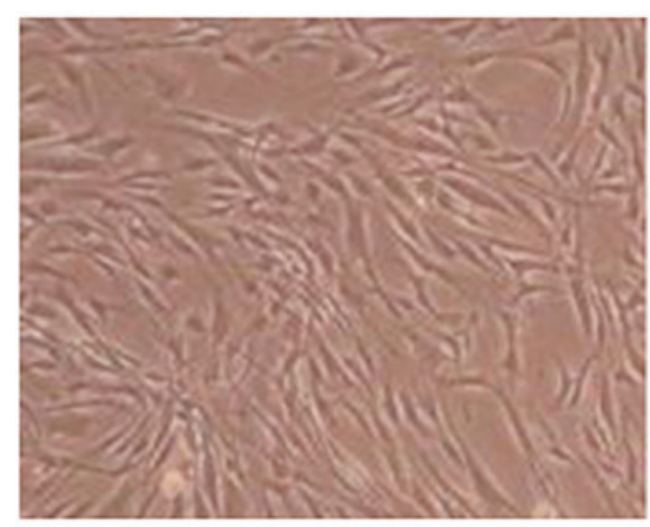

Figure 1. Cell morphology. Cell morphology of OLF cell. Magnification, $\mathrm{x} 20$. The cells remained shuttle shape until the end of week 1 . OLF cell morphology was observed after 3 weeks mechanical stress.

in the culture flasks. In addition, the cells exhibited a shuttle shape until the end of week 1 (Fig. 1).

Cell viability. When comparing the viability of OLF cells among the different experimental groups, the control and unstretched groups demonstrated similar levels of cell viability at 1,2 and 3 weeks (Fig. 2). However, mechanical stress significantly increased OLF cell viability following $2(\mathrm{P}<0.05)$ and 3 weeks $(\mathrm{P}<0.01)$ of mechanical stimulation when compared with the controls (Fig. 2).

Osteogenic differentiation. Oil Red O staining was performed to analyze the effect of 3 weeks of mechanical stress on the osteogenic differentiation of OLF cells. The osteogenic differentiation rate of the control group appeared to be similar to that of the unstretched group (Fig. 3). By contrast, mechanical stress effectively promoted the osteogenic differentiation rate and increased the size of OLF cells, when compared with the control group (Fig. 3).

Expression of osteocalcin (OC), alkaline phosphatase (ALP) and runt-related transcription factor 2 (RUNX-2) $m R N A$. RT-qPCR analysis was performed to investigate OC, ALP and RUNX-2 mRNA expression levels following mechanical

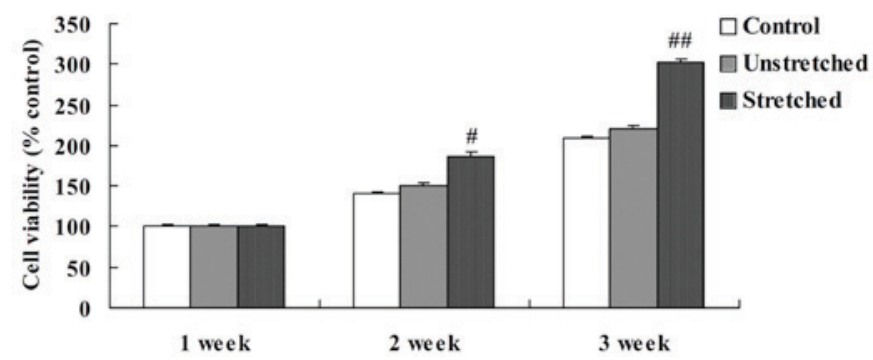

Figure 2. The percentage viability of cells derived from patients with ossification of the ligamentum flavumin the control, unstretched and stretched groups following 1,2 and 3 weeks of mechanical stress. ${ }^{~} \mathrm{P}<0.05$ and ${ }^{\# \#} \mathrm{P}<0.01$ vs. the control group. Control, untreated control group; unstretched, non-mechanical stress group; stretched, mechanical stress group.

stress stimulation at 3 weeks. As shown in Fig. 4, the mRNA expression levels of OC, ALP and RUNX-2 were comparable when comparing the control group and unstretched groups. By contrast, mechanical stress significantly increased the expression of OC, ALP and RUNX-2 mRNA in OLF cells when compared with the control group ( $\mathrm{P}<0.01 ;$ Fig. 4).

Expression of Ets proto-oncogene 1 (Ets-1) and sex determining region $Y$-box 2 (SOX-2) $m R N A$. In order to investigate the mechanisms underlying the effects of mechanical stress on osteogenic OLF cell differentiation, Ets-1 and SOX-2 mRNA expression was determined by RT-qPCR analysis. Following quantitative analysis, no significant difference in Ets-1 and SOX-2 miRNA expression levels were observed between the control and unstretched groups (Fig. 5). However, mechanical stress significantly inhibited Ets-1 and SOX-2 mRNA expression in OLF cells when compared with the control cells $(\mathrm{P}<0.01$; Fig. 5).

Expression of Src protein. Western blot analysis revealed that the control and unstretched groups exhibited similar levels of Src protein expression (Fig. 6). By contrast, mechanical stress significantly inhibited Src protein expression in OLF cells when compared with the control group $(\mathrm{P}<0.01$; Fig. 6$)$.

Expression of p-p38MAPK protein. To further elucidate the underlying mechanisms of mechanical stress on osteogenic OLF cell differentiation, p-p38MAPK protein was detected 
using western blot analysis. No significant differences inp-p38MAPK protein expression were observed between the control and unstretched groups (Fig. 7). However, mechanical stress significantly increased p-p38MAPK protein expression, when compared with the control group $(\mathrm{P}<0.01$; Fig. 7).

Expression of BMP protein. BMP protein expression was determined by western blot analysis. As shown in Fig. 8, no significant difference in BMP protein expression was observed between the control and unstretched groups. However, mechanical stress significantly increased BMP protein expression in OLF cells when compared with the control group $(\mathrm{P}<0.01$; Fig. 8).

Expression of Smad-1 protein. p-Smad-1 protein expression in OLF cells was analyzed using western blot analysis. As shown in Fig. 9, no significant difference in the protein expression of p-Smad-1 was observed between the control and unstretched groups. However, mechanical stress significantly increased p-Smad-1 protein expression in OLF cells, when compared with the control group $(\mathrm{P}<0.01$; Fig. 9).

\section{Discussion}

Following the action of mechanical stress stimuli, bone tissues transform mechanical stress signals into local signals, which target stress-sensitive cells via the integrating integrin-cytoskeleton system, the calcium channel pathway and the primary cilium pathways (16). Stress-sensitive cells transform these primary biochemical signals into downstream signals through a signal transduction channel (6). These signals subsequently induce alterations in gene expression, energy metabolism and material synthesis. In the present study, mechanical stress effectively increased cell viability and promoted the osteogenic differentiation rate of OLF cells. Mechanical stimuli induce alterations in bone microstructure and produce a number of local signals, which are recognized by mechanical stress-sensitive cells (17). Li et al (18) demonstrated that mechanical strain regulates osteogenic differentiation in bone marrow stromal cells (BMSCs).

An ongoing study showed the complex underlying mechanisms of signal transduction are currently unclear and are therefore the focus of a study (19). The process by which cells produce extracellular chemical energy from mechanical energy is of particular interest $(19,20)$. Previous studies have demonstrated that cyclicalmechanical stress is regulated by kinases to promote the proliferation of chondrocytes by functioning as extracellular signaling molecules $(19,21)$. In the present study, mechanical stress significantly increased OC, ALP and RUNX-2 mRNA expression levels, while significantly inhibiting Ets-1 and SOX-2 miRNA expression in OLF cells. Li et al (18) demonstrated that mechanical strain regulates osteogenic differentiation in BMSCs via Runx2, Osterix, and collagen-I.

Src, a member of the Src family, possesses tyrosine protein kinase activity. Phosphorylation of the Tyr residue on the activation loop activates specific enzymes and the delivery of extracellular signals to the cells, thereby facilitating cell proliferation and migration (22). A previous study demonstrated that the migration activity of murine fibroblast

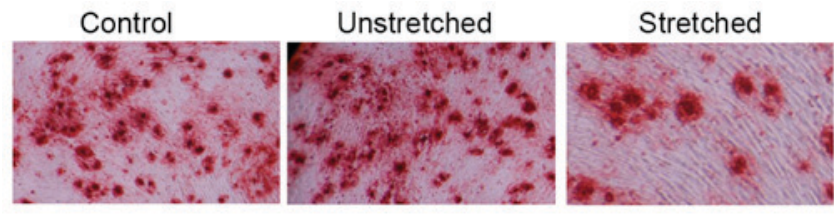

Figure 3. Osteogenic differentiation. Representative images of Oil Red O-stained ossification of the ligamentum flavum cells in the control, unstretched, and stretched groups (magnification x20). Control, untreated control group; unstretched, non-mechanical stress group; stretched, mechanical stress group.
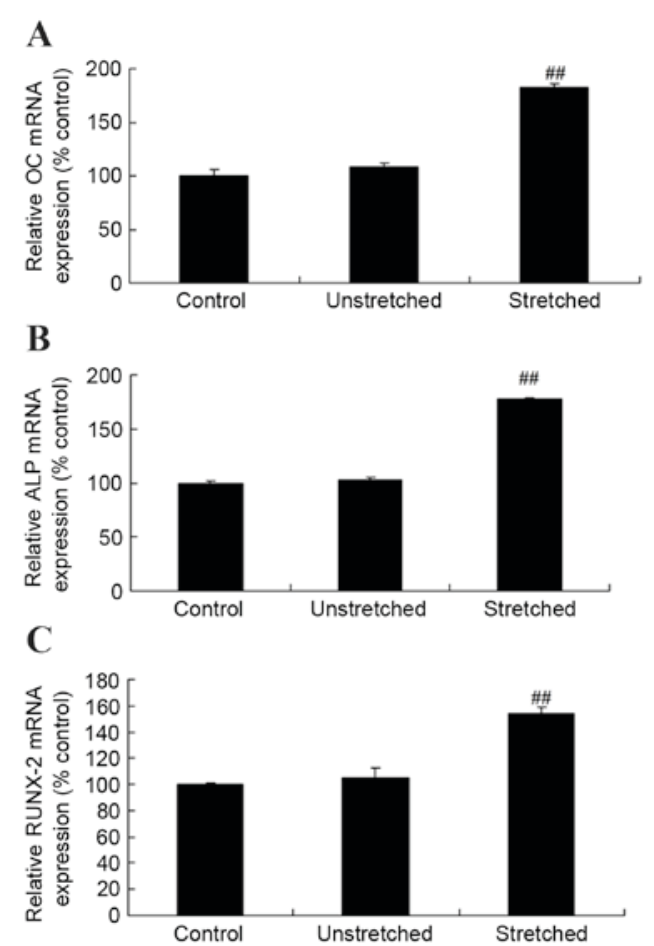

Figure 4. Expression of (A) OC, (B) ALP and (C) RUNX-2 mRNA in ossification of ligamentum flavum cells following mechanical stress, as determined by reverse transcription-quantitative polymerase chain reaction analysis. ${ }^{\# \prime} \mathrm{P}<0.01$ vs. control group. OC, osteocalcin; ALP, alkaline phosphatase; RUNX-2, runt-related transcription factor 2; control, untreated control group; unstretched, non-mechanical stress group; stretched, mechanical stress group.

cells in Src-knockout rats is reduced, which further supports the role of $\mathrm{Src}$ in cell migration (22). The interactions between Src, the extracellular matrix and integrin are essential for the induction of ras homolog gene family, member A (RhoA), and in turn, the activation of RhoA is closely associated with cell migration (23). The present study revealed that mechanical stress significantly suppressed Src protein expression in OLF cells. Paravicini et al (24) demonstrated that mechanical stretch influences vascular fibrosis during hypertension, which appeared to be independent of c-Src and p38MAPK.

p38MAPK is a core component of the MAPK cascade. Phosphorylation of p38MAPKinduces enzyme activation and serves a fundamental role in cell proliferation, differentiation and migration (25). p38MAPK at different cellular locations possess different functions. p38MAPK on the cytomembrane 
A

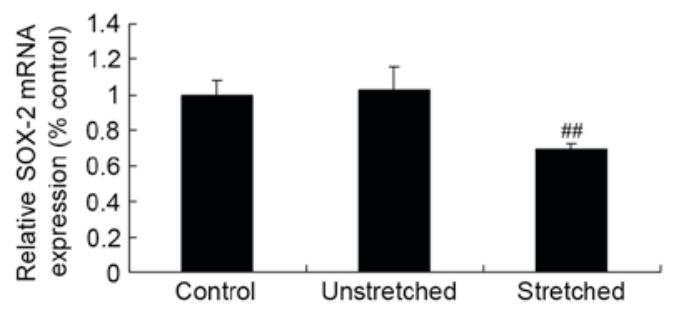

B

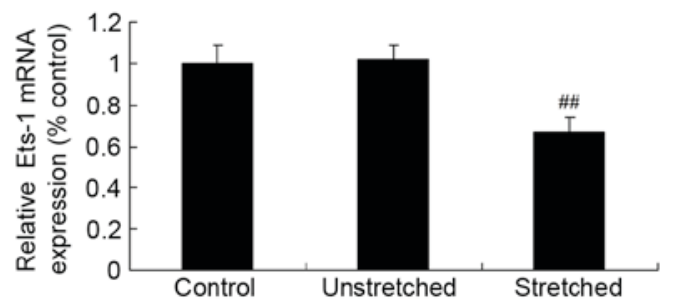

Figure 5. Expression of (A) Ets-1 and (B) SOX-2 mRNA in ossification of ligamentum flavum cells following mechanical stress as determined by reverse transcription-quantitative polymerase chain reaction analysis. ${ }^{\#} \mathrm{P}<0.01$ vs. control group. Ets-1, Ets proto-oncogene 1 ; SOX-2, sex determining region Y-box 2; control, untreated control group; unstretched, non-mechanical stress group; stretched, mechanical stress group.

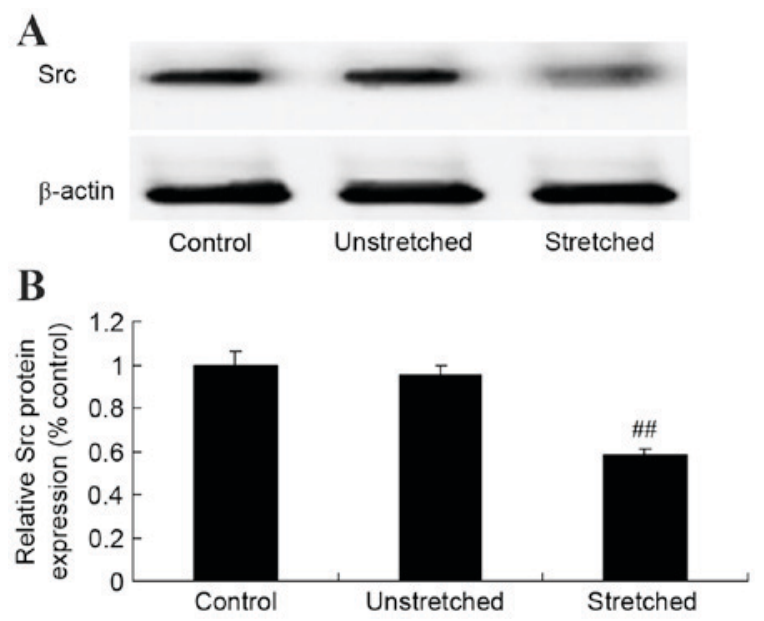

Figure 6. Expression of Src protein in ossification of the ligamentum flavum cells following mechanical stress. (A) Western blotting analysis of Src protein expressionand (B) quantification of Src protein expression levels following normalization to $\beta$-actin expression. ${ }^{\#} \mathrm{P}<0.01$ vs. control group. Control, untreated control group; unstretched, non-mechanical stress group; stretched, mechanical stress group.

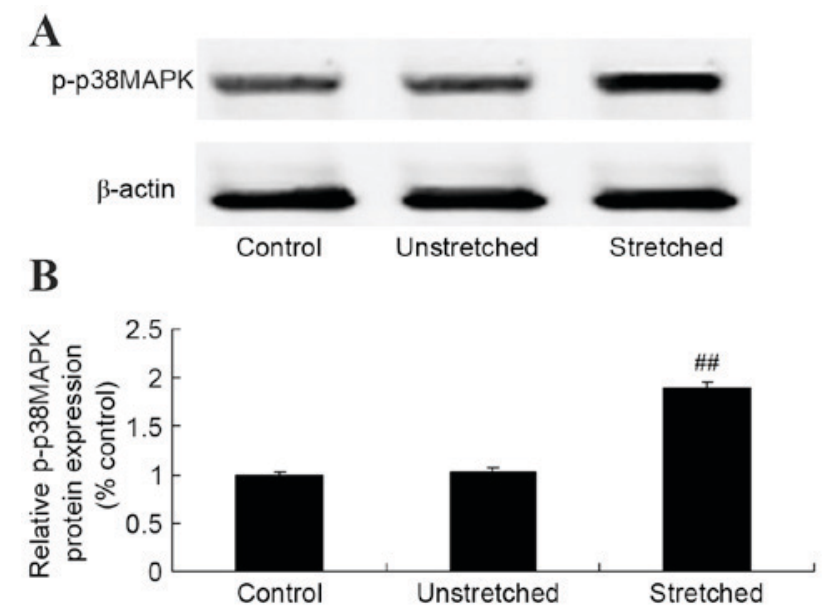

Figure 7. Expression of p-p38MAPK protein by (A) western blotting analysis, and (B) quantification of p-p38MAPK protein expression following normalization to $\beta$-actin expression. ${ }^{\# \#} \mathrm{P}<0.01$ vs. control group. p38MAPK, p38-mitogen-activated protein kinase; p-p38MAPK phosphorylated p38MAPK; control, untreated control group; unstretched, non-mechanical stress group; stretched, mechanical stress group.

participates in cellular effects, and following mechanical trauma, induces the migration and differentiation of stomach
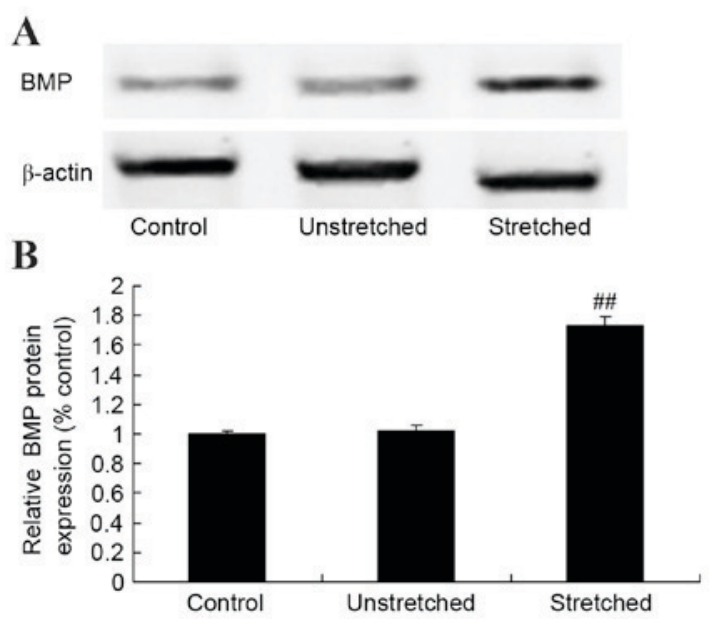

Figure 8. Expression of BMP protein in ossification of the ligamentum flavum cells. The expression of BMP protein by (A) western blotting analysis and (B) quantification of BMP protein expression following normalization to $\beta$-actin expression. ${ }^{\# \#} \mathrm{P}<0.01$ vs. control group. BMP, bone morphogenetic protein; control, untreated control group; unstretched, non-mechanical stress group; stretched, mechanical stress group.

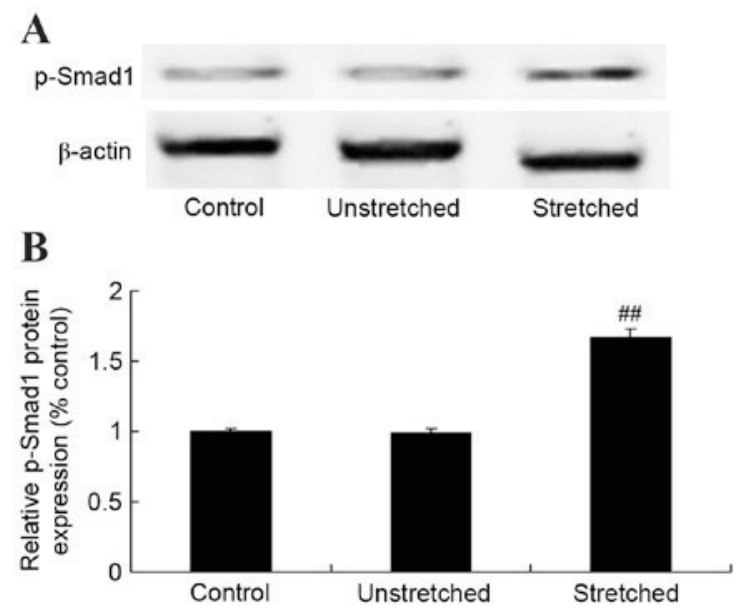

Figure 9. Expression of p-Smad-1 protein in ossification of the ligamentum flavum cells by (A) western blotting analysis and (B) quantification of p-Smad-1 protein expression following normalization to $\beta$-actin expression. ${ }^{\# \#} \mathrm{P}<0.01$ vs. control group. p-SMAD-1, phosphorylated-mothers against decapentaplegic homolog-1; control, untreated control group; unstretched, non-mechanical stress group; stretched, mechanical stress group.

smooth muscle cells (26). This function does not depend on the intranuclear transcription. The present study demonstrated 
that mechanical stress significantly increased p-p38MAPK protein expression in OLF cells.

A study confirmed that the BMP-Smad-1 signaling pathway regulates specific aspects of the osteoblast life cycle, including differentiation from mesenchymal stem cells to osteoblasts, osteogenic cell proliferation, the vitality of osteoblast mineralization and osteoclast coupling (27). However, abnormal differentiation is triggered by downregulated Runx2 and Osterix expression (28). Empirical data collected from a recent study indicated that the loss of BMP-Smad signaling significantly increased Osterix and Runx 2 protein expression $(29,30)$. The results of the present study demonstrated that mechanical stress significantly increased BMP and p-Smad1 protein expression in OLF cells. Kido et al (31) revealed that mechanical stress activates the Smad pathway in osteoblasts. In addition, Grottkau et al (32) demonstrated that mechanical stretching induced osteogenesis through BMP-2 mRNA expression in adipose-derived stem cells and BMSCs.

In conclusion, the present study demonstrated that mechanical stress effectively increased cell viability and promoted the osteogenic differentiation rate of OLF cells potentially via the activation of OC, ALP and RUNX-2, and the suppression of Ets-1 and SOX-2 signaling. These results suggest that the BMP-Smad-1 signaling pathway may serve an important role in mediating the intracellular signaling effects of mechanical stress on osteogenic differentiation via the Src and p38MAPK signaling pathways in OLF cells.

\section{References}

1. Yamazaki M, Koda M, Okawa A and Aiba A: Transient paraparesis after laminectomy for thoracic ossification of the posterior longitudinal ligament and ossification of the ligamentum flavum. Spinal Cord 44: 130-134, 2006.

2. Dorenbeck U, Schreyer AG, Schlaier J, Held P, Feuerbach S and Seitz J: Degenerative diseases of the cervical spine: Comparison of a multiecho data image combination sequence with a magnetisation transfer saturation pulse and cervical myelography and CT. Neuroradiology 46: 306-309, 2004.

3. Yabe Y, Hagiwara Y, Tsuchiya M, Honda M, Hatori K, Sonofuchi K, Kanazawa K, Koide M, Sekiguchi T, Itaya N and Itoi E: Decreased elastic fibers and increased proteoglycans in the ligamentum flavum of patients with lumbar spinal canal stenosis. J Orthop Res 34: 1241-1247, 2016.

4. Wang T, Pan M, Yin CQ, Zheng XJ, Cong YN, Wang DC and Li SZ: Spinal cord kinking in thoracic myelopathy caused by ossification of the ligamentum flavum. Chin Med J (Engl) 128: 2595-2598, 2015.

5. Li S, Xia H and Han C: Retrospective analysis on correlation factors of preserving the ligamentum flavum in microendoscopic discectomy. Clin Neurol Neurosurg 139: 46-50, 2015.

6. Chiang $\mathrm{CW}$, Chen WC, Liu HW, Wang IC and Chen $\mathrm{CH}$ : Evaluating osteogenic potential of ligamentum flavum cells cultivated in photoresponsive hydrogel that incorporates bone morphogenetic protein-2 for spinal fusion. Int J Mol Sci 16: 23318-23336, 2015.

7. Tani T, Kawasaki M, Taniguchi S and Ushida T: Functional importance of degenerative spondylolisthesis in cervical spondylotic myelopathy in the elderly. Spine (Phila Pa 1976) 28: $1128-1134,2003$

8. Oliva A, Llabrés M and Fariña JB: Fitting bevacizumab aggregation kinetic data with the Finke-Watzky two-step model: Effect of thermal and mechanical stress. Eur J Pharm Sci 77: 170-179, 2015.

9. Kameo Y and Adachi T: Interstitial fluid flow in canaliculi as a mechanical stimulus for cancellous bone remodeling: In silico validation. Biomech Model Mechanobiol 13: 851-860, 2014.
10. Mander L, Wesseln CJ,McElwain JC and Punyasena SW: Tracking taphonomic regimes using chemical and mechanical damage of pollen and spores: An example from the Triassic-Jurassic mass extinction. PLoS One 7: e49153, 2012.

11. Cremers NA, Suttorp M, Gerritsen MM, Wong RJ, van Run-van Breda C, van Dam GM, Brouwer KM, Kuijpers-Jagtman AM, Carels CE, Lundvig DM and Wagener FA: Mechanical stress changes the complex interplay between HO-1, inflammation and fibrosis, during excisional wound repair. Front Med (Lausanne) 2: 86, 2015.

12. Li WJ, Guo SG, Sun ZJ and Zhao Y: Multilevel thoracic ossification of ligamentum flavum coexisted with/without lumbar spinal stenosis: Staged surgical strategy and clinical outcomes. BMC Musculoskelet Disord 16: 206, 2015.

13. Inoue H, Seichi A, Kimura A, Endo T and Hoshino Y: Multiple-level ossification of the ligamentum flavum in the cervical spine combined with calcification of the cervical ligamentum flavum and posterior atlanto-axial membrane. Eur Spine J 22 (Suppl 3): S416-S420, 2013.

14. Qi MC, Hu J, Zou SJ, Chen HQ, Zhou HX and Han LC: Mechanical strain induces osteogenic differentiation: Cbfal and Ets-1 expression in stretched rat mesenchymal stem cells. Int J Oral Maxillofac Surg 37: 453-458, 2008.

15. Livak KJ and Schmittgen TD: Analysis of relative gene expression data using real-time quantitative PCR and the 2(-Delta Delta C(T)) method. Methods 25: 402-408, 2001.

16. Zhu Z, Fu Y, Tian D, Sun N, Han W, Chang G, Dong Y, Xu X, Liu Q, Huang D and Shi FD: Combination of the immune modulator fingolimod with alteplase in acute ischemic stroke: A pilot trial. Circulation 132: 1104-1112, 2015.

17. Fal K, Landrein B and Hamant O: Interplay between miRNA regulation and mechanical stress for CUC gene expression at the shoot apical meristem. Plant Signal Behav 11: e1127497, 2016.

18. Li R, Liang L, Dou Y, Huang Z, Mo H, Wang Y and Yu B: Mechanical strain regulates osteogenic and adipogenic differentiation of bone marrow mesenchymal stem cells. Biomed Res Int 2015: 873251, 2015.

19. Akimoto T, Okuhira K, Aizawa K, Wada S, Honda H, Fukubayashi $\mathrm{T}$ and Ushida T: Skeletal muscle adaptation in response to mechanical stress in p130 $\mathrm{cas}^{-/-}$mice. Am J Physiol Cell Physiol 304: C541-C547, 2013.

20. Uyttewaal M,Burian A,Alim K, Landrein B, Borowska-Wykret D, Dedieu A, Peaucelle A, Ludynia M, Traas J, Boudaoud A, et al: Mechanical stress acts via katanin to amplify differences in growth rate between adjacent cells in Arabidopsis. Cell 149: 439-451, 2012.

21. Li H, Zhang XY, Wu TJ, Cheng W, Liu X, Jiang TT, Wen J, Li J, Ma QL and Hua ZC: Endoplasmic reticulum stress regulates rat mandibular cartilage thinning under compressive mechanical stress. J Biol Chem 288: 18172-18183, 2013.

22. Chong YP, Ia KK, Mulhern TD and Cheng HC: Endogenous and synthetic inhibitors of the Src-family protein tyrosine kinases. Biochim Biophys Acta 1754: 210-220, 2005.

23. Marton N, Baricza E, Érsek B, Buzás EI and Nagy G: The emerging and diverse roles of Src-like adaptor proteins in health and disease. Mediators Inflamm 2015: 952536, 2015.

24. Paravicini TM, Montezano AC, Yusuf H and Touyz RM: Activation of vascular p38MAPK by mechanical stretch is independent of c-Src and NADPH oxidase: Influence of hypertension and angiotensin II. J Am Soc Hypertens 6: 169-178, 2012.

25. Li Y, Tao J, Zhang J, Tian X, Liu S, Sun M, Zhang X, Yan C and Han Y: Cellular repressor E1A-stimulated genes controls phenotypic switching of adventitial fibroblasts by blocking p38MAPK activation. Atherosclerosis 225: 304-314, 2012.

26. Xiao WL, Zhang DZ, Fan CH and Yu BJ: Intermittent stretching and osteogenic differentiation of bone marrow derived mesenchymal stem cells via the p38MAPK-Osterix signaling pathway. Cell Physiol Biochem 36: 1015-1025, 2015.

27. Matsumoto Y, Otsuka F, Takano M, Mukai T, Yamanaka R, Takeda M, Miyoshi T, Inagaki K, Sada KE and Makino H: Estrogen and glucocorticoid regulate osteoblast differentiation through the interaction of bone morphogenetic protein-2 and tumor necrosis factor-alpha in C2C12 cells. Mol Cell Endocrinol 325: 118-127, 2010.

28. Susperregui AR, Viñals F, Ho PW, Gillespie MT, Martin TJ and Ventura F: BMP-2 regulation of PTHrP and osteoclastogenic factors during osteoblast differentiation of $\mathrm{C} 2 \mathrm{C} 12$ cells. J Cell Physiol 216: 144-152, 2008. 
29. Okamoto M, Murai J, Yoshikawa $\mathrm{H}$ and Tsumaki N: Bone morphogenetic proteins in bone stimulate osteoclasts and osteoblasts during bone development. J Bone Miner Res 21: 1022-1033, 2006.

30. Ghosh-Choudhury N, Singha PK, Woodruff K, St Clair P, Bsoul S, Werner SL and Choudhury GG: Concerted action of Smad and CREB-binding protein regulates bone morphogenetic protein-2-stimulated osteoblastic colony-stimulating factor-1 expression. J Biol Chem 281: 20160-20170, 2006.
31. Kido S, Kuriwaka-Kido R, Umino-Miyatani Y, Endo I, Inoue D, Taniguchi H, Inoue Y, Imamura T and Matsumoto T: Mechanical stress activates Smad pathway through PKCס to enhance interleukin-11 gene transcription in osteoblasts. PLoS One 5: pii: e13090, 2010.

32. Grottkau BE, Yang X, Zhang L, Ye L and Lin Y: Comparison of effects of mechanical stretching on osteogenic potential of ASCs and BMSCs. Bone Res 1: 282-290, 2013. 